\title{
Full Waveform Inversion of Multi-shot Seismic Data Acquired for Complex Subsurface Structure
}

\author{
Ehsan Jamali Hondori, Hitoshi Mikada, Tada-nori Goto, and Junichi Takekawa \\ Dept. of Civil and Earth Res. Eng., Kyoto University
}

\begin{abstract}
We studied full waveform inversion of seismic data acquired in elastic media to develop subsurface images from raw shot gathers. The forward modeling is based on finite difference solution of the elastic wave equation in the frequency domain. Gradient vector is calculated using an adjoint-state technique and pseudo Hessian matrix is used to precondition the gradient vector to update the model parameter via preconditioned conjugate gradient method. Two synthetic examples from crosswell tomography and surface acquisition experiments are presented to examine the ability of the method in proper subsurface imaging.
\end{abstract}

\section{INTRODUCTION}

Full Waveform Inversion (FWI) is becoming a reliable tool to develop high resolution subsurface images from seismic data. In contrast to travel time tomography, which only uses first arrival times for inversion, FWI considers all kind of the events in the waveforms including direct arrivals, reflections, refractions, multiples, and surface waves. This provides more information for the misfit evaluation to find an optimized seismic model which matches the recorded data. Full waveform inversion may be applied in time domain ${ }^{1,2)}$ or in frequency domain ${ }^{3,4)}$. In frequency domain, the inversion problem can be solved for increasing frequencies from lower to higher values. By this technique the low wavenumber images are reconstructed first and then high wavenumber images from higher frequency components of the wavefields increase the resolution of the subsurface model. This multiscale strategy helps to overcome the nonlinearity of the problem. Also, once triangular factors of impedance matrix have been calculated multiple sources can be processed with the minimum computational costs. Monochromatic and band limited inversion at desired frequencies are possible and the attenuation behavior of the media can directly be dealt with considering complex valued elastic parameters. We used finite difference method ${ }^{5)}$ to develop our forward model for FWI in frequency domain. In order to suppress the edge reflections and the corner diffractions, we used Perfectly Matched Layers $\left.{ }^{6}\right)$ attached to the boundaries of the computational area. The full waveform inversion problem is solved by an iterative local optimization approach based on a steepest-descent method. The gradient of the objective function is computed using the adjoint-state method which allows avoiding the explicit computation of the sensitivity matrix. A preconditioned conjugate gradient method, using a pseudo Hessian ${ }^{7)}$ as a preconditioner, iteratively updates the model parameters. The final model of the current iteration is used as the initial model for the subsequent iteration and the inversion of each frequency group starts with the latest model from previous frequency group. Results of a crosswell tomography experiment and surface acquisition provide good images from subsurface.

\section{THE FORWARD PROBLEM}

One can write $2 \mathrm{D}$ elastic wave equation in frequency domain as

$$
\begin{aligned}
& \omega^{2} \rho u+\frac{\partial}{\partial x}\left[\lambda\left(\frac{\partial u}{\partial x}+\frac{\partial v}{\partial z}\right)+2 \mu \frac{\partial u}{\partial x}\right] \\
& +\frac{\partial}{\partial z}\left[\mu\left(\frac{\partial v}{\partial x}+\frac{\partial u}{\partial z}\right)\right]+f_{x}=0 \\
& \omega^{2} \rho v+\frac{\partial}{\partial z}\left[\lambda\left(\frac{\partial u}{\partial x}+\frac{\partial v}{\partial z}\right)+2 \mu \frac{\partial v}{\partial z}\right] \\
& +\frac{\partial}{\partial x}\left[\mu\left(\frac{\partial v}{\partial x}+\frac{\partial u}{\partial z}\right)\right]+f_{z}=0
\end{aligned}
$$

where, $u, v, \omega, \rho, \lambda$, and $\mu$ are horizontal and vertical displacements, angular frequency, bulk density, and Lame parameters, respectively. $f_{x}$ and $f_{z}$ are horizontal and vertical external forces. We used 25-point finite difference weighted averaging stencil $^{5)}$ to discretize the wave equation. In a grid of 
size $n \times n$, equation (1) can be rewritten in matrix from as

$$
\mathrm{Su}=\mathrm{f}
$$

where, $\mathrm{S}$ is the complex impedance matrix with size of $2 n^{2} \times 2 n^{2}$, u and $\mathrm{f}$ are displacement and source vectors, respectively. In order to solve the system of equations one may factorize impedance matrix $\mathrm{S}$ and then use forward/backward substitution. The impedance matrix has a sparse pattern and it is important to use efficient ordering schemes to reduce fill-in during LU factorization. We used a nested dissection strategy to speed up our LU factorization. Reflections from truncated boundaries appear in the solutions of the wave equation which must be suppressed adequately. Perfectly Matched Layers $^{6}$ found to be the most effective method to eliminate boundary reflections and we used this technique to suppress the edge reflections.

\section{THE INVERSION PROBLEM}

Full waveform inversion in frequency domain has been well illustrated in previous studies ${ }^{3,8)}$. The problem is solved via local optimization algorithms; global optimization methods are not feasible because of the large number of model parameters. The cost function is defined as the least squares norm of residuals, i.e. the difference between recorded data and calculated model, as

$$
E(p)=\frac{1}{2} \Delta \mathrm{d}^{t} \Delta \mathrm{d}^{*}
$$

where, $\Delta \mathrm{d}$ is the residual vector and superscripts $t$ and * denote transpose and complex conjugate, respectively. The model parameter $p$ is updated by preconditioned conjugate gradient method ${ }^{9)}$ and gradient vector can be efficiently calculated by adjoint-state method without explicitly forming the computational demanding sensitivity matrix. Full waveform inversion is accomplished via a multiscale scheme from lower to higher frequencies to overcome nonlinearity of the inversion problem. Frequencies are divided into different frequency groups and frequencies in each group can be inverted either simultaneously or sequentially. The resulting model from each group is used as starting model for the next group. The preconditioned gradient with respect to model parameter $p_{i}$ at iteration $k$ is

$$
\mathrm{g}_{k, p_{i}}=\operatorname{diag}(\mathrm{H}+\gamma \mathrm{I})^{-1} \operatorname{Real}\left[\mathrm{u}^{t} \frac{\partial \mathrm{S}^{t}}{\partial p_{i}} \mathrm{~S}^{-1} \Delta \mathrm{d}^{*}\right]
$$

where, $\mathrm{H}$ and $\mathrm{I}$ are the diagonal pseudo Hessian ${ }^{7)}$ and identity matrix, respectively. $\gamma$ is damping factor and $\mathrm{u}$ is the model vector of horizontal and vertical displacements. Time reversed, i.e. complex conjugated in frequency domain, residuals are backpropagated from receiver locations and multiply with forward model to calculate gradient vector. The model parameter $p$ is updated as

$$
\mathrm{p}_{k+1}=\mathrm{p}_{k}+\alpha \delta_{k}
$$

where, step length $\alpha$ must be small enough to achieve accurate results without trapping in a local minimum of the cost function. This parameter can be calculated by linear approximations or line search methods. The conjugate direction and scaling are defined as

$$
\begin{aligned}
& \delta_{k}=-\mathrm{g}_{k}+\beta_{k} \delta_{k-1} \\
& \beta_{k}=\frac{\left\langle g_{k}, g_{k}-g_{k-1}\right\rangle}{\left\|g_{k-1}\right\|^{2}}
\end{aligned}
$$

The model parameter is updated in a direction which is defined by a combination of gradient at iteration $k$ and update direction at iteration $k-1$. This will help the algorithm to converge in a small number of iterations.

\section{SYNTHETIC EXAMPLES}

\subsection{FWI Tomography}

A crosswell full waveform tomography simulation is conducted to make subsurface images from a model which includes different typical shapes. True $\mathrm{P}$ wave velocity model is shown in Figure 1. Density is assumed to be fixed at 2200 $\mathrm{kg} / \mathrm{m}^{3}$ and will be known during the inversion. Total number of 27 explosive sources are placed in the left well at $50 \mathrm{~m}$ intervals and 140 receivers are located in the right well with $10 \mathrm{~m}$ intervals. The source wavelet is a Ricker wavelet with dominant frequency of $30 \mathrm{~Hz}$ and maximum recorded and inverted frequency is $60 \mathrm{~Hz}$. All the frequencies are inverted in a multiscale scheme in 50 iterations. Six frequency groups have been inverted from lower to higher values and each group includes 12 frequencies which have been inverted simultaneously. The final frequency in each group is repeated in the next frequency group to provide an overlapping band between the groups. 

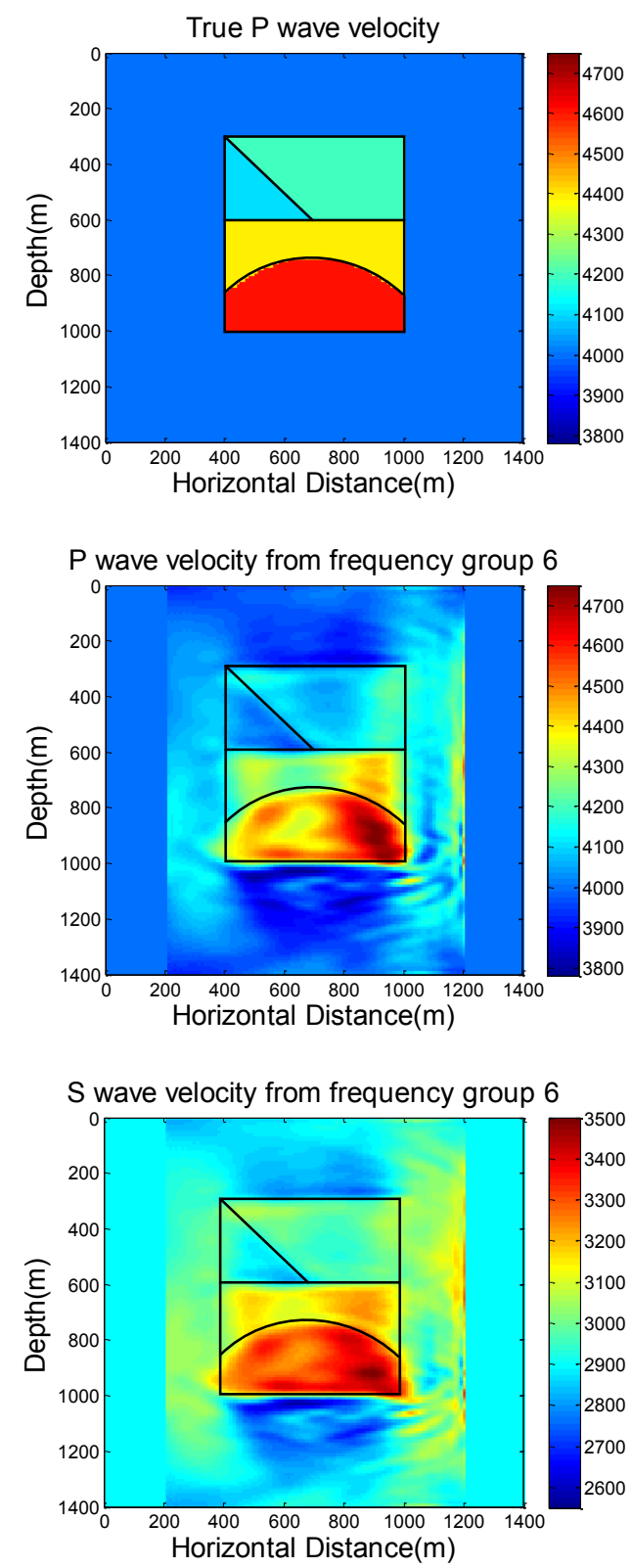

Figure 1 True $\mathrm{P}$ wave velocity, and resulting $\mathrm{P}$ and $\mathrm{S}$ wave velocity models.
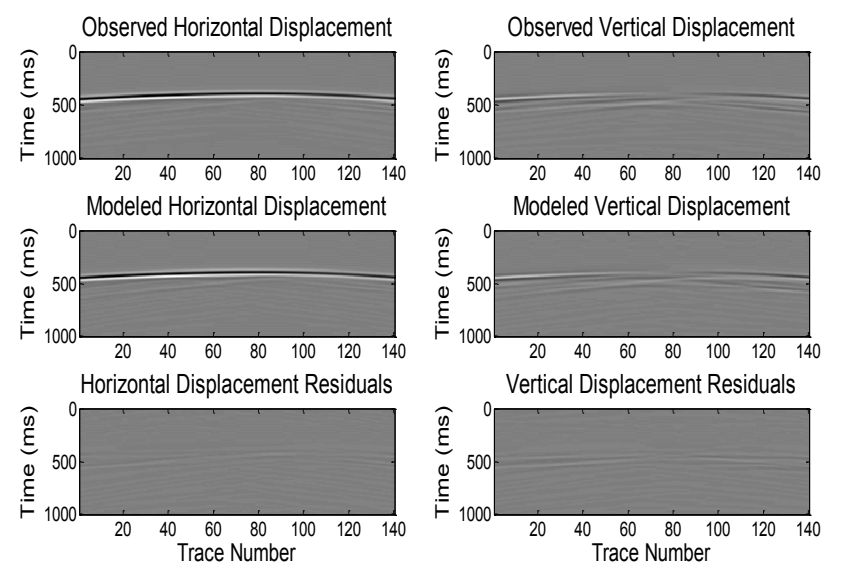

Figure 2 Observed, modeled, and residual horizontal and vertical displacement wavefields.
The background velocity field is used as the initial velocity model. Lame parameters are inverted simultaneously and $\mathrm{P}$ and $\mathrm{S}$ wave velocities are calculated after achieving final results. We didn't update the model parameters near source and receiver locations to stay away from source singularities and the high amplitudes in the gradient vector at these locations. The final velocity models after inverting all the frequency groups are shown in Figure 1. Both $\mathrm{P}$ and $\mathrm{S}$ wave velocity models could successfully resolve different parts of the true model. The observed data, calculated model and residual wavefields resulting from a single shot gather are shown in Figure 2. All the figures have same color scale. Small amplitudes of the residuals confirm that the resulted images are very close to the true model.

\subsection{FWI of Seismic Reflection Data}

In the second example (Figure 3) we applied full waveform inversion on synthetic seismic reflection data. The true model which is $1000 \mathrm{~m}$ long and $500 \mathrm{~m}$ deep includes three distinct layers. Two component geophones are located on the surface at every 25 meters. Seismic sources are located $50 \mathrm{~m}$ below the surface at every 25 meters. Only ten discrete frequencies have been used for full waveform inversion ${ }^{10)}$ with two different strategies. First we tried to invert all the frequencies simultaneously. The result of this simultaneous inversion is shown in Figure 3. The initial model is a linear interpolation of the true model from top to bottom. We didn't update the model parameters in the top layer to get rid of source and receiver high amplitudes. Three layers could be successfully detected by the algorithm and the velocities are in good accordance with the true model. In the second attempt we used three overlapping frequency groups and the inversion started from lower frequencies to higher values. Each group included 4 frequency components and an overlapping frequency is repeated in groups (Table 1). The frequencies in each group are inverted simultaneously and the result of the current group is used as the starting model for the next group. All three layers could be recovered in 20 iterations and the velocities are much closer to the true model, compared to the first strategy.

Table 1 Frequency groups for inversion.

\begin{tabular}{|c|c|}
\hline Frequency Group & Frequency Components \\
\hline 1 & $2,2.77,3.84,5.32$ \\
\hline 2 & $5.32,7.38,10.23,14.19$ \\
\hline 3 & $14.19,19.67,27.26,37.80$ \\
\hline
\end{tabular}




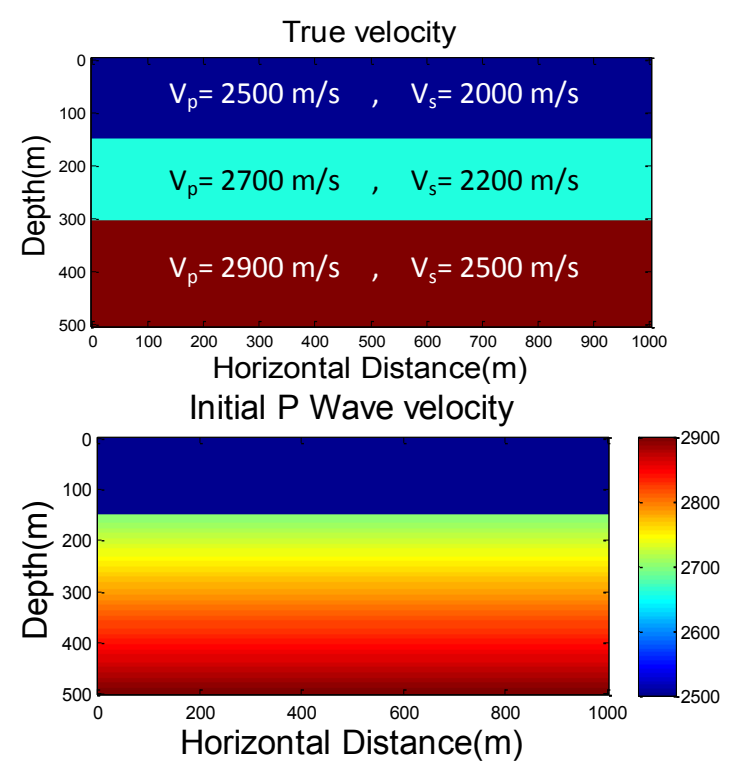

$P$ wave velocity from Simultaneous Inversion

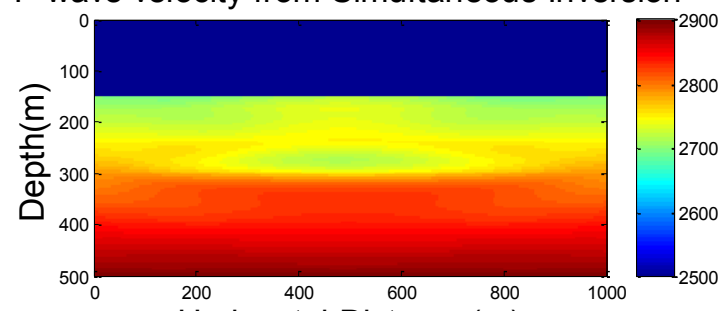

Horizontal Distance $(\mathrm{m})$

$S$ wave velocity from Simultaneous Inversion

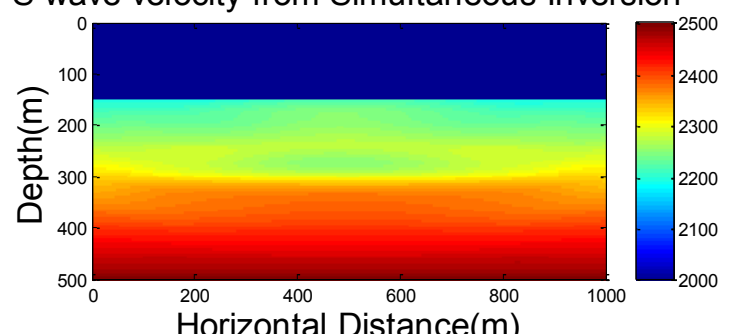

Horizontal Distance $(\mathrm{m})$

$\mathrm{P}$ wave velocity from frequency group 3

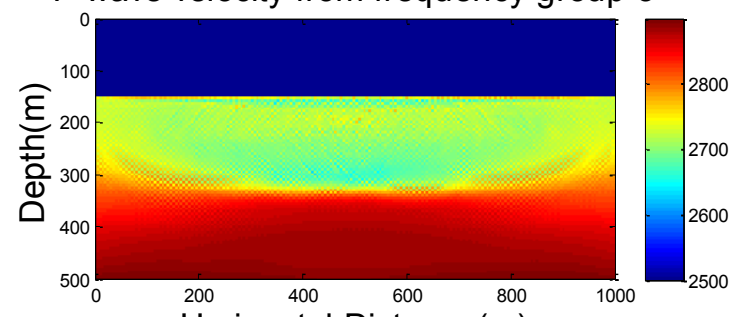

Horizontal Distance $(\mathrm{m})$

$\mathrm{S}$ wave velocity from frequency group 3

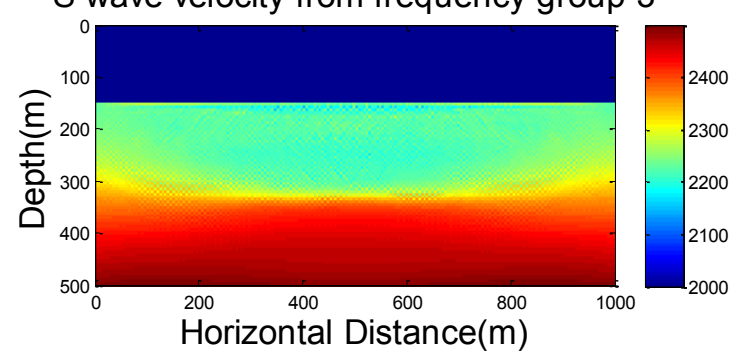

Figure 1 True velocity model, initial model, resulting P and $\mathrm{S}$ wave velocity from simultaneous inversion, and sequential inversion of frequency groups.

\section{Conclusions}

Seismic full waveform inversion has been studied to develop subsurface images. By inverting different frequency groups from low to high frequencies, the nonlinearity of the inversion problem is mitigated. A preconditioned conjugate gradient method has been used to iteratively update the model parameter. The gradient of the cost function is calculated by adjoint-state method without explicitly forming the sensitivity matrix. Crosswell full waveform tomography results could detect the subsurface anomalies with good accuracy. Full waveform inversion of seismic reflection data could detect the layers and the velocity amplitudes. Sequential inversion of overlapping frequency groups provides better results compared to simultaneous inversion of all the frequencies.

\section{REFERENCES}

1) Tarantola, A., 1984, Inversion of seismic reflection data in the acoustic approximation, Geophysics, 49, 1259-1266.

2) Mora, P. R., 1987, Nonlinear two-dimensional elastic inversion of multi-offset seismic data, Geophysics, 52, 1211-1228

3) Pratt, R. G., C. Shin, and G. J. Hicks, 1998, Gauss-Newton and full Newton methods in frequency-space seismic waveform inversion, Geophysical Journal International, 133, 341-362.

4) Brossier, R., S. Operto, and J. Virieux, 2009a, Seismic imaging of complex structures by $2 \mathrm{D}$ elastic frequency-domain full-waveform inversion, Geophysics, 74 (6), WCC63-WCC76.

5) Min, D., Shin, C., Kwon, B. and Chung, S., 2000, Improved frequency-domain elastic wave modeling using weighted-averaging difference operators, Geophysics, 65, 3, 884-895.

6) Berenger, J. P., 1994, A perfectly matched layer for absorption of electromagnetic waves, Journal of Computational Physics, 114, 185-200.

7) Shin, C., S. Jang, and D. J. Min, 2001, Improved amplitude preservation for prestack depth migration by inverse scattering theory, Geophysical Prospecting, 49, 592-606.

8) Virieux , J., and Operto, S., 2009, An overview of full-waveform inversion in exploration geophysics;, Geophysics, 74, WCC1-WCC26.

9) Vigh, D., and E. W. Starr, 2008a, 3D plane-wave full-waveform inversion, Geophysics, 73, (5), VE135-VE144.

10) Sirgue, L., and R. G. Pratt, 2004, Efficient waveform inversion and imaging: A strategy for selecting temporal frequencies, Geophysics, 69, $231-248$ 